\title{
Las ciudades medias de España y sus coronas en el siglo XXI (2000-2017): dinámica demográfica y desarrollo inmobiliario
}

As cidades médias da Espanha e suas coroas no século XXI (2000-2017): dinâmica demográfica e desenvolvimento imobiliário

Medium-sized cities of Spain and their crowns in the 21 st century (2000-2017): population and real estate dynamics

José María Martínez Navarro[a] (D), Juan Antonio García González[b] (D),

Luis Alfonso Escudero Gómez[c] (1)

[a] Universidad Autónoma de Madrid (UAM), Facultad de Filosofía y Letras, Madrid, España

[b] Universidad de Castilla-La Mancha (UCLM), Facultad de Humanidades, Albacete, España

[c] Universidad de Castilla-La Mancha (UCLM), Facultad de Humanidades, Toledo, España

Cómo citar: Martínez Navarro, J. M., García González, J. A., \& Escudero Gómez, L. A. (2020). Las ciudades medias de España y sus coronas en el siglo XXI (2000-2017): dinámica demográfica y desarrollo inmobiliario. urbe. Revista Brasileira de Gestão Urbana, 12, e20190202. https://doi.org/10.1590/2175-3369.012.e20190202

\section{Resumen}

El inicio del siglo XXI ha sido muy compulsivo en la dinámica urbana española. La causa principal ha sido el crecimiento urbanístico explosivo que tuvo lugar entre 2000 y 2008 y su siguiente paralización por la Gran Recesión. El artículo analiza este proceso centrado en las ciudades medias y sus respectivas coronas dentro del contexto nacional. Se plantea un estudio comparado de la evolución de la población y de las unidades constructivas de las ciudades medias y sus áreas urbanas. Las fuentes utilizadas son el Padrón Municipal de Habitantes y el Catastro Inmobiliario. La investigación se realiza mediante un método deductivo. Los resultados obtenidos muestran una respuesta dispar de las ciudades españolas dentro del patrón común marcado por el punto de inflexión que supuso la crisis, con un contexto general de desarrollo hasta 2008 y de caída posterior. Además, se produce un comportamiento diferente del crecimiento urbano en relación con la proximidad a una gran área metropolitana.

Palabras clave: Geografía urbana. Ciudades medias. Periferias urbanas. Áreas metropolitanas. Dinámicas urbanas. España.

\section{Resumo}

$O$ início do século XXI tem sido muito compulsivo para a dinâmica urbana espanhola. A principal causa se deve ao crescimento urbano explosivo que ocorreu entre 2000 e 2008 e a consequente estagnação por causa da Grande Recessão. O artigo analisa esse processo centrado em cidades de médio porte e suas respectivas coroas dentro do contexto nacional. É apresentado um estudo comparativo da evolução da população e das unidades construtivas em cidades de médio porte e suas áreas urbanas. As fontes utilizadas são o Registro Municipal de Habitantes e o Cadastro Predial. A pesquisa é realizada por meio do método dedutivo. Os resultados obtidos mostram uma resposta distinta entre as cidades espanholas de acordo com o padrão comum marcado pelo ponto de inflexão 
da crise, com um contexto geral de desenvolvimento até 2008 e queda subsequente. Além disso, há um comportamento diferente do crescimento urbano em relação à proximidade com uma grande área metropolitana.

Palavras-chave: Geografia urbana. Cidades médias. Periferias urbanas. Áreas metropolitanas. Dinâmica urbana. Espanha.

\section{Abstract}

The beginning of the 21st century was very compulsive concerning to the Spanish urban dynamic. The main cause has been the explosive urban growth that took place between 2000 and 2008 and its next stoppage by the Great Recession. The article analyses this process centred on medium-sized cities and their respective crowns within the national context. A comparative study of the evolution of the population and the constructive units in medium-sized cities and their urban areas was presented. The sources used are the Municipal Register of Inhabitants and the Real Estate Cadastre. The research is carried out by a deductive method. The results obtained show a disparate response of Spanish cities within the common pattern marked by the tipping point of the crisis, with a general context of growth until 2008 and subsequent fall. In addition, there is a different behaviour of urban growth in relation to proximity to a large metropolitan area.

Keywords: Urban geography. Medium-sized cities. Urban peripheries. Urban system. Urban dynamics. Spain.

\section{Introducción}

Los seres humanos que han sido reducidos a la condición de seres urbanos. (Galeano, 1998, p. 243).

Las ciudades medias han sido, de forma general, centros regionales que han actuado como mercado; han producido y, sobre todo, distribuido bienes y servicios; han organizado la red de infraestructuras viarias y de transporte y, muy frecuentemente, han administrado una demarcación política que en España ha sido la provincia (Ganau \& Vilagrasa, 2003). Las nuevas lógicas de urbanización aparecidas en las últimas décadas en estas ciudades han alterado su tradicional estructura compacta, intensa y densa. Han dado paso a nuevas formas territoriales más dispersas acompañadas de transformaciones morfológicas, funcionales y sociales (Bellet \& Gutiérrez, 2015). Se trata de unos cambios que se corresponden al modelo de "ciudad difusa" según explica Indovina (1998).

Desde 1980 y fundamentalmente entre 1990 y hasta la crisis de 2008, se ha producido en España un intenso proceso de urbanización dispersa que ha afectado a todo el país. Se trata de dinámicas recientes relacionadas con el boom inmobiliario y la creciente artificialización del suelo (Fernández \& Corbelle, 2017). Son las áreas urbanas intermedias las que experimentan los mayores incrementos relativos, muy por encima de los que presentan las principales aglomeraciones urbanas (Bellet \& Olazábal, 2017). Además, se han originado ciudades supramunicipales generadas por la suma de fragmentos sin continuidad en su tejido urbano (Zúñiga, 2016). Así se ha difundido tanto el fenómeno urbano, como las redes de urbanización en la práctica totalidad del territorio (Roca et al., 2012), con lo que, consecuentemente, se ha cambiado el rol y la dinámica territorial de estos núcleos. Con todos estos factores, se ha generado una fragmentación espacial y social y una especialización funcional en las ciudades medias (Bellet, 2012).

Este artículo se plantea participar en el debate sobre las dinámicas actuales de urbanización en el entorno de las ciudades medias. Hildreth (2006) señala que hay una relativa falta de publicaciones sobre núcleos urbanos medianos comparada con la amplitud del material sobre ciudades grandes. Sin embargo, actualmente las ciudades medias han ganado importancia en el campo de la investigación urbana (Adam, 2006). De hecho, Gutiérrez \& Paül (2012) han realizado una selección y categorización de la bibliografía académica publicada en todo el mundo referida al estudio de las ciudades intermedias que así lo demuestra. 
En los últimos años han proliferado estudios sobre ciudades medias dentro de los campos de investigación sobre análisis urbano. Este interés aparece en trabajos de grandes conjuntos regionales mundiales, principalmente en ciudades medias americanas (Maturana \& Rojas, 2016) y europeas (Servillo et al., 2017). También son frecuentes las investigaciones que, como la presente, se centran en una escala nacional; por ejemplo, Obrębalski (2017) sobre las ciudades medias de la República Checa; Moyart (2005) acerca de las de Francia; o Marques da Costa (2002), centrándose en las portuguesas. Em muchos casos son estudios difícilmente extrapolables ya que el tamaño de dichas ciudades, así como su área de influencia territorial varía considerablemente. Sistemas urbanos diferentes con elementos diversos y geometrías variables abren un amplio abanico de posibilidades empezando por el tamaño de las ciudades medias (Pérez \& Rojas, 2017; Angeoletto et al., 2016).

Las publicaciones sobre ciudades medias españolas se han multiplicado en los últimos años, aunque tiene lugar un debate sobre la cuestión desde hace ya casi cuatro décadas (Campos Sánchez, 2017). Como antecedente, autores como Abellán et al. (1978), ya realizaron en el año 1978 una propuesta de tipología de ciudades españolas de tipo medio. El mayor número de publicaciones se ha realizado desde la Geografía (Cano, 2008). Han aparecido libros como el de Precedo \& Míguez (2014) sobre las ciudades medias españolas dentro del contexto de la globalización; investigaciones en congresos como la de Vilagrasa (1999) que aborda las ciudades pequeñas y medias de España o las comunicaciones de la primera ponencia del XIII Congreso de la Población Española, Metropolitanización: procesos y estructuras demográfico-territoriales, editadas por Reques \& De Cos (2012) donde se analizan diferentes dinámicas urbanas en múltiples ciudades medias de España; capítulos de libros como el de Méndez (2010) que estudia el significado actual de las ciudades intermedias y sus periferias; y, por supuesto, artículos científicos como el de López Trigal (2014) que identifica y caracteriza estratégicamente las ciudades medias de España y Portugal. Además, han aparecido investigaciones específicas referidas a comunidades autónomas (Cebrián, 2007) o a urbes concretas (Llorente del Río, 2010).

No es sencillo delimitar las ciudades medias de España como área de estudio ni tan siquiera definir qué es una ciudad media. Las definiciones de ciudad media, o de ciudad intermedia, difieren en el entorno español y en el mundo. Se ha llegado a poner en cuestión la verdadera necesidad de una definición acabada sobre este fenómeno complejo y con límites difusos (Andrés, 2008). Para algunos autores, se trata de núcleos que actúan como nexo entre niveles urbanos superiores e inferiores y establecen relaciones con otras ciudades y territorios del mismo nivel jerárquico (Garrido-Jiménez et al., 2017). Para otros, actúan como intermediarios entre la gran ciudad y los espacios rurales (Vilagrasa, 2000).

El número de habitantes ha sido el factor más recurrente para hacerlo. Se trata de una perspectiva pragmática y cuantitativa de delimitación de las ciudades medias (Adam, 2006). La ciudad media sería aquella que no es ni pequeña ni grande (Marinovic-Uzelac, 1999). En realidad, el tamaño de las ciudades medias varía con la escala de análisis (Marques da Costa, 2002). Existen importantes diferencias y no resulta posible utilizar los mismos rangos cuantitativos para definir las ciudades medias en diferentes contextos (Garrido-Jiménez et al., 2017).

Incluso, no hay una definición homogénea en el ámbito español y sí un cierto grado de arbitrariedad (Campos Sánchez, 2017). Es necesario continuar investigando las ciudades medias para intentar definirlas y dimensionarlas (Llop \& Usón, 2012). Diversos autores han establecido unos límites poblacionales distintos para las ciudades medias españolas (ver Tabla 1).

Tabla 1 - Delimitaciones poblacionales de las ciudades medias dentro de la literatura española

\begin{tabular}{cc}
\hline Límites de habitantes de las ciudades medias & Referencias bibliográficas \\
\hline $20.000-100.000$ & Esteban \& López (1989) \\
$20.000-200.000$ & Vilagrasa (1999) \\
$20.000-250.000$ & Méndez (2010) \\
$20.000-300.000$ & Somoza Medina (2013) \\
$50.000-100.000$ & Vinuesa (1989) \\
$50.000-200.000$ & Salom (1995) \\
\hline
\end{tabular}


Tabla 1 - Continuação...

\begin{tabular}{|c|c|}
\hline Límites de habitantes de las ciudades medias & Referencias bibliográficas \\
\hline $50.000-250.000$ & $\begin{array}{c}\text { Andrés (2008); Escudero Gomez \& Somoza Medina } \\
\text { (2010); Bellet \& Olazábal (2017) }\end{array}$ \\
\hline $50.000-300.000 *$ & Mallarach y Vilagrasa (2002) y Ganau \& Vilagrasa (2003) \\
\hline $50.000-500.000$ & López Trigal (2014) \\
\hline
\end{tabular}

*Límites poblacionales aplicados en el presente artículo. Fuente: Autores (2019).

El análisis que se presenta sigue los mismos criterios que en el trabajo de Mallarach \& Vilagrasa (2002) y el de Ganau \& Vilagrasa (2003), quienes, partiendo de los 50.000 mil habitantes, consideran que los 300.000 residentes es un tope superior ajustado al contexto urbano español para delimitar estos núcleos intermedios.

No obstante, la noción de una ciudad media no es rígida y no puede estar únicamente condicionada por el tamaño (Andrés, 2008). Cualquier horquilla poblacional resultará incompleta. La diversidad de valores propuestos en diferentes estudios demuestra la insuficiencia del criterio exclusivamente poblacional (Marques da Costa, 2002). Por ello, se ha decidido incluir en el análisis a aquellas capitales de provincia con más de 300.000 habitantes (Zaragoza, Palma, Córdoba y Valladolid) o con menos de 50.000 habitantes (Soria y Teruel) localizadas en provincias donde no haya ninguna ciudad media. Se considera la relevancia de estas capitales de provincia como ciudades intermedias - al igual que hacen Bellet \& Olazábal (2017) para las menores-y que son estos núcleos urbanos los que, realmente, asumen la funcionalidad propia de las ciudades medias de manera independiente a su tamaño demográfico. Finalmente, el estudio se basa en 136 ciudades medias.

El área de estudio se completa con una corona de municipios aledaños a cada una de estas ciudades con el fin de descubrir los efectos de la urbanización difusa en sus entornos inmediatos. Con este objetivo, era necesario delimitar algún criterio para establecer la periferia posible a partir de la ciudad media central. Nuevamente, se decide fijar un límite arbitrario de partida: un radio de 30 kilómetros desde el núcleo principal para definir las áreas urbanas intermedias siguiendo los trabajos anteriores de Mallarach \& Vilagrasa (2002) y de Adam (2006). La simple distancia euclidiana es el punto de salida, pero no puede ser la respuesta a la definición de las áreas urbanas de las ciudades medias; no obstante, es un primer elemento de acercamiento

Este criterio se completa con otro demográfico al prescindir en la muestra de todos los municipios rurales de menos de 10.000 habitantes que presentan una dinámica demográfica negativa entre 2000 y 2016. La pérdida de población constante durante todo el periodo estudiado muestra la ausencia de una influencia de difusión urbana. Finalmente, el estudio incluye un total de 2.028 municipios alrededor de las ciudades medias (ver Figura 1). 


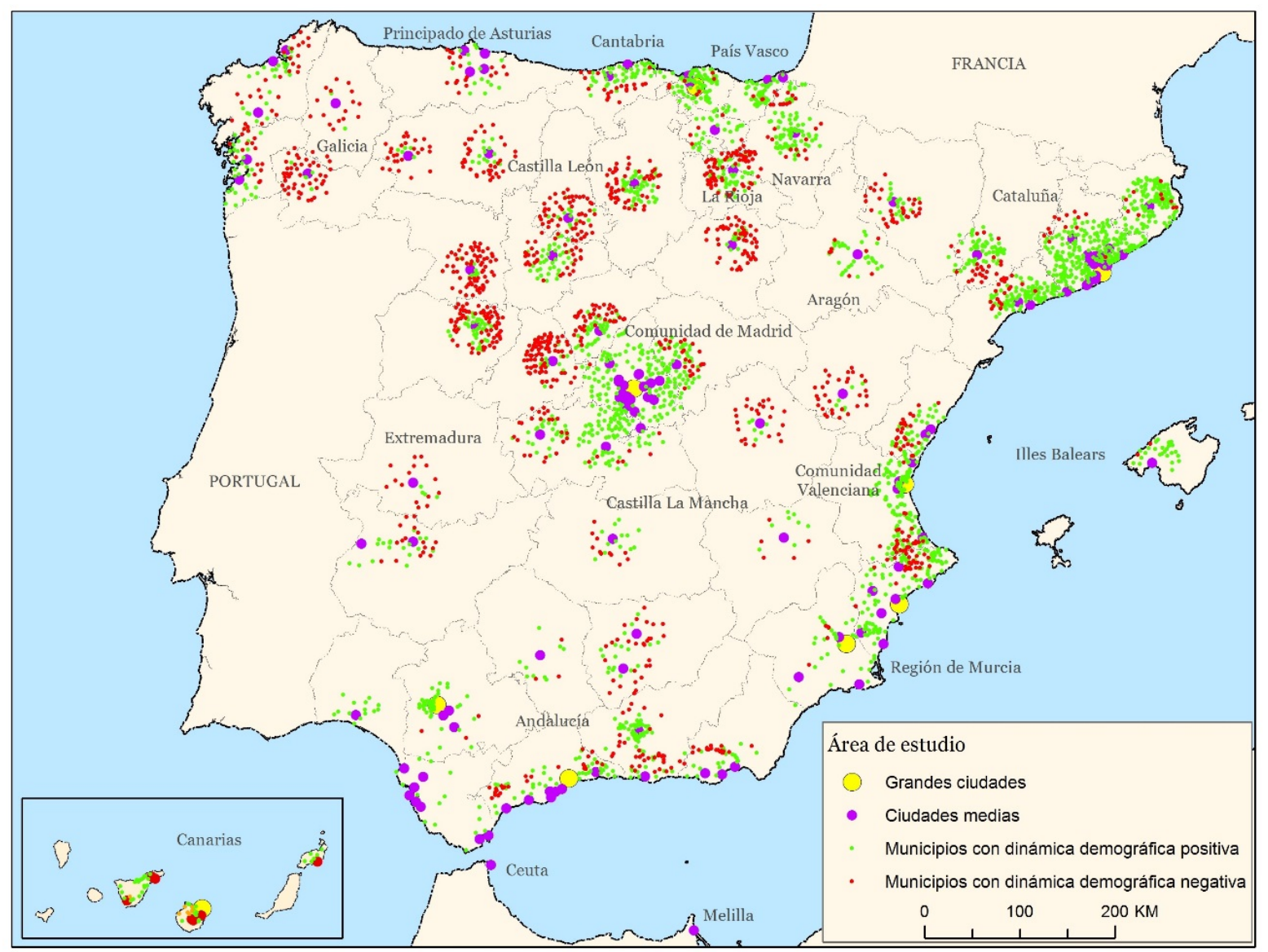

Figura 1 - Delimitación del área de estudio. Fuente: Autores (2019).

Por otro lado, es indudable el importante papel que tienen en el sistema urbano español las ciudades grandes (Feria \& Albertos, 2010). La evolución de estos grandes núcleos urbanos ha creado grandes áreas metropolitanas, claves en la organización territorial y socioeconómica de España en la actualidad. En este estudio no participan directamente nueve de las trece ciudades grandes (ver Tabla 2), pero su influencia y presencia surge a partir de las ciudades medias metropolitanas. Son varias las ciudades medias ubicadas en el entorno metropolitano de las grandes urbes españolas y cuya dinámica no cabe duda de que viene condicionada por dicha ciudad grande (De Ureña et al., 2013).

Tabla 2 - Delimitaciones poblacionales de las ciudades medias dentro de la literatura española

\begin{tabular}{ccccc}
\hline Número de municipios & $\begin{array}{c}\text { Muestra } \\
\text { ciudades } \\
\text { medias* }\end{array}$ & $\begin{array}{c}\text { Coronas } \\
\text { ciudades } \\
\text { medias }\end{array}$ & $\begin{array}{c}\text { Resto de } \\
\text { municipios }\end{array}$ & $\begin{array}{c}\text { Total } \\
\text { nacional }\end{array}$ \\
\hline $\begin{array}{c}\text { 1 Grandes ciudades (<300.000 hab.) } \\
\text { 2 Ciudades medias (De 50.000 a 299.999 hab.) }\end{array}$ & 4 & & 9 & 13 \\
3 Ciudades pequeñas (De 10.000 a 49.999 hab.) & 2 & 433 & 171 & 130 \\
4 Mun. rurales +1000 hab. & & 974 & 1439 & 2413 \\
5 Mun. rurales -1000 hab. & $\mathbf{1 3 6}$ & 621 & 4327 & 4948 \\
Total general & $\mathbf{2 0 2 8}$ & $\mathbf{5 9 3 7}$ & $\mathbf{8 1 1 0}$ \\
\hline
\end{tabular}

*Incluye Zaragoza, Palma, Córdoba, Valladolid, Soria y Teruel. Fuente: Padrón Municipal de Habitantes de 2016 (INE, 2018 ).

El objetivo principal del trabajo es analizar los diferentes comportamientos de las ciudades medias del sistema urbano español comparando el crecimiento demográfico con su comportamiento urbanístico en dos periodos de tiempo sucesivos bien diferenciados. Se pretende aportar una comprensión al debate actual sobre la dinámica y comportamiento de las ciudades medias en España. 


\section{Metodología}

La investigación está realizada mediante un método deductivo y el análisis de los datos utilizados ha sido estadístico y cartográfico. Se ha optado por el uso de dos fuentes cuantitativas para desarrollar la parte empírica de la investigación: el Padrón Municipal de Habitantes (INE, 2018) y el Catastro Inmobiliario (Espanha, 2018).

El Padrón Municipal de Habitantes es una de las fuentes de información demográfica de referencia en España Al tratarse de una fuente anual, facilita la investigación de la evolución poblacional (Clayton \& Morris, 2010). De hecho, permite establecer dos periodos de análisis que van desde el 2000 al 2008 y desde el 2009 hasta el 2016. Presenta algunas irregularidades como sobrestimar el total de la población de muchos municipios (Reher \& Valero Lobo, 2005), aunque se vienen haciendo procesos constantes de mejora de este registro demográfico.

La segunda fuente utilizada son las estadísticas oficiales del Catastro Inmobiliario de la Dirección General del Catastro, dependiente del Ministerio de Hacienda. Son datos de carácter fiscal, a partir de los cuales se calcula el importe del impuesto municipal de bienes inmuebles, pero con una gran relevancia para realizar investigaciones urbanas como demuestra Pillet (2008). Son datos perfectamente útiles para analizar la evolución constructiva ya que todas las edificaciones registradas tienen asociada una fecha de construcción. La Dirección General del Catastro (Espanha, 2018) resume el número de unidades de construcción nuevas a escala municipal por décadas y hasta el presente. Así, se pueden extraer fácilmente los inmuebles existentes en cada municipio que han sido construidos desde el año 2000 hasta el 2009 o desde el año 2010 hasta la actualidad. Esto supone un pequeño desajuste con respecto a los periodos de análisis que utilizamos para la demografía, pero no se considera un problema metodológico porque la fecha del catastro es la de inscripción, es decir, cuando el edificio en cuestión está terminado y no cuando se inicia su promoción. El problema principal de los datos del catastro es geográfico. No hay datos de las comunidades autónomas del País Vasco y Navarra al tener transferidas las competencias en materia fiscal y no son datos públicos. por ello quedan fuera del estudio de la dinámica constructiva de Baracaldo, Guecho, Irún, Pamplona, San Sebastián, Vitoria y sus coronas.

\section{La dinámica poblacional de las ciudades medias de España y sus coronas en el siglo XXI}

Se analiza la evolución de la población en el periodo comprendido entre el año 2000 y el 2016, teniendo en cuenta al año 2008 como punto de inflexión, al producirse el inicio de la Gran Recesión. Los resultados obtenidos son reflejados en la Tabla 3.

Durante el periodo del denominado boom (2000-2008), todas las unidades territoriales crecen destacando los municipios de las coronas de las ciudades medias que duplican el crecimiento de estas. Por el contrario, entre 2008 y 2016 aparece una situación de estancamiento demográfico, aunque las ciudades medias, y en mayor medida sus coronas, siguen ganando población, al revés que el resto del territorio (ver Tabla 3).

Tabla 3 - Evolución de la población por categoría de análisis propuesta

\begin{tabular}{|c|c|c|c|c|c|c|}
\hline & \multicolumn{2}{|c|}{ Padrón 2000} & \multicolumn{2}{|c|}{ Padrón 2008} & \multicolumn{2}{|c|}{ Padrón 2016} \\
\hline & Total & $\%$ & Total & $\%$ & Total & $\%$ \\
\hline Muestra ciudades medias & 13.956 .561 & $34 \%$ & 15.822 .569 & $34 \%$ & 16.065 .837 & $34 \%$ \\
\hline Coronas ciudades medias & 10.043 .814 & $25 \%$ & 12.696 .087 & $27 \%$ & 13.361 .381 & $29 \%$ \\
\hline Resto de municipios & 16.596 .562 & $41 \%$ & 17.765 .317 & $38 \%$ & 17.234 .676 & $37 \%$ \\
\hline Total & 40.596 .937 & $100 \%$ & 46.283 .973 & $100 \%$ & 46.661 .894 & $100 \%$ \\
\hline
\end{tabular}

Fuente: Padrón de Municipal de Habitantes (INE, 2018). 
El peso porcentual de la población de las ciudades medias españolas se mantiene a lo largo de todo el periodo, incrementándose en un $4 \%$ el volumen poblacional de sus coronas a costa de la pérdida del resto de municipios, incluyendo a las nueve grandes ciudades.

A partir de esta evolución (ver Tabla 4), los municipios de las coronas de las ciudades medias acumulan, en el padrón de 2016, aproximadamente el 30\% de la población total de España. Es un porcentaje ligeramente inferior a la suma de los habitantes de todas las ciudades medias $(35,43 \%)$, pero 12 puntos porcentuales por encima de la población que residen en las grandes ciudades $(17,83 \%)$. Fuera de la influencia urbana de grandes y medias ciudades se localizan el 73\% de los municipios españoles, 171 de ellos son urbanos, pero únicamente vive el 16,7\% de la población española. En definitiva, y en números redondos, 64 de cada 100 españoles reside en una ciudad media o su corona, incluyendo las excepciones descritas en su categorización, 18 en una gran ciudad y tan solo 17 en un municipio inferior a 50.000 habitantes, cuyo núcleo principal está a más de 30 kilómetros de una ciudad media.

Tabla 4 - Delimitaciones poblacionales de las ciudades medias dentro de la literatura española

\begin{tabular}{ccc}
\hline \% de crecimiento en período & $\mathbf{2 0 0 0 / 2 0 0 8}$ & $\mathbf{2 0 0 9 / 2 0 1 6}$ \\
\hline Muestra ciudades medias & $13 \%$ & $2 \%$ \\
Coronas ciudades medias & $26 \%$ & $5 \%$ \\
Resto de municipios & $7 \%$ & $-3 \%$ \\
\hline
\end{tabular}

Fuente: Padrón de Municipal de Habitantes (INE, 2018).

En la Figura 2 se cartografía la evolución de la población de las ciudades medias y sus coronas en el periodo expansivo, 2000-2008. La primera lectura que se obtiene es la mancha de crecimientos fuertes representados en verdes: son valores sostenidos por encima del 0,5\% en las periferias de Madrid y Barcelona y en el Mediterráneo, con especial relevancia en la costa andaluza y en la Costa Blanca alicantina. De manera general son tres zonas las que destacan por los aumentos demográficos en España en este periodo: el Levante mediterráneo, el valle del Ebro y el área central de Madrid. Por otro lado, la dispersión de puntos del mapa refleja los resultados obtenidos en la Tabla 3, de tal forma que las coronas de las ciudades medias experimentan un incremento superior de población entre 2000-2008.

En las ciudades medias, los ascensos son moderados, por debajo del $0,5 \%$, mientras que en los municipios colindantes, más pequeños, hay incrementos de incluso el $15 \%$. Al trabajar con datos porcentuales, los cuales permiten una comparación entre municipios de distinto tamaño demográfico, es más factible obtener porcentajes de crecimiento más alto en municipios de pequeño tamaño. 


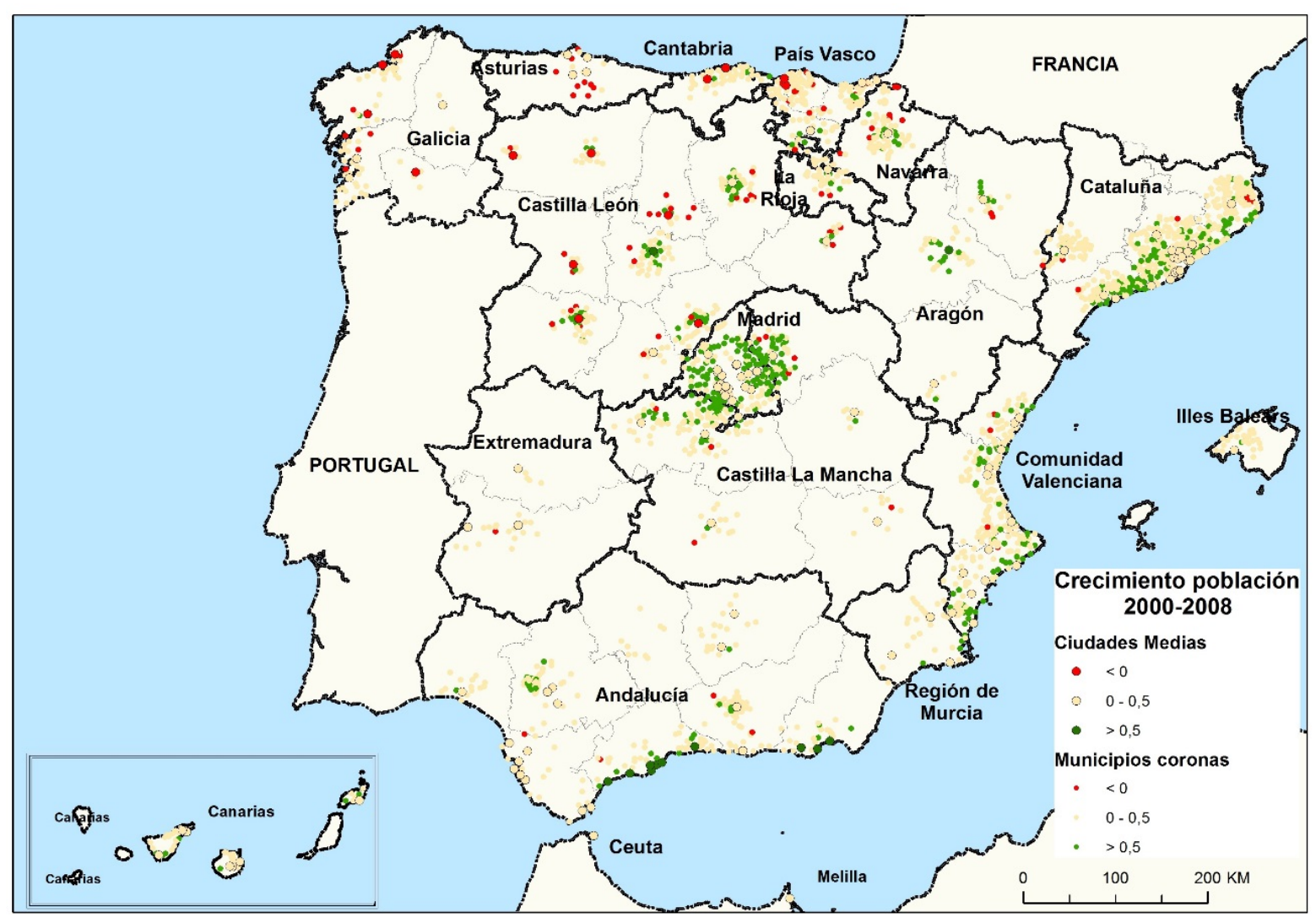

Figura 2 - Evolución de la población en las ciudades medias y sus coronas entre 2000 y 2008. Fuente: Padrón Municipal de Habitantes (INE, 2018) y autores (2019).

Los crecimientos negativos son puntuales, salvo en el sector noroccidental, donde pierden población las ciudades medias de Avilés, Ferrol, Guecho, León, Orense, Salamanca, Santander-Torrelavega (dos ciudades cántabras que forman un espacio de cohesión como área metropolitana de tamaño medio según De Cos \& De Meer, 2013) y Valladolid.

Por lo tanto, aparece un claro contraste en la evolución de las ciudades medias de España y sus coronas entre el norte y oeste de España y el centro peninsular. Como ocurre de forma significativa, tanto en las ciudades medias y las áreas urbanas próximas a Madrid y también en el Mediterráneo, así como en los dos archipiélagos. Los grandes crecimientos demográficos del periodo tienen lugar en estas últimas zonas.

En la Figura 3 se cartografía la evolución de la población en la etapa de recesión económica, comprendida entre 2008 y 2016. Para ello, se ha utilizado la misma fuente, los mismos intervalos y el mismo sistema de representación. El cambio es drástico. Los colores verdes, los valores más altos de crecimiento demográfico, han desaparecido prácticamente. Se aprecia un decrecimiento paulatino desde las ciudades medias centrales hacia los municipios más lejanos de sus coronas. En la dispersión de puntos también se comprueba una mayor resistencia en los municipios de Madrid y Barcelona con incrementos La disminución del crecimiento del total de habitantes se extiende a todas las ciudades medias y a sus respectivas coronas; es decir, la paralización poblacional es evidente. Así se demuestra en el norte y occidente de España, pero también se constata en espacios anteriormente muy dinámicos. Es el caso del área metropolitana madrileña y sus influencias más externas en las provincias de otras comunidades que la rodean. También destaca la reducción del crecimiento poblacional en el ámbito mediterráneo frente al anterior aumento demográfico de esta zona en los primeros ocho años del siglo XXI y en décadas anteriores (Gaja, 2008). 


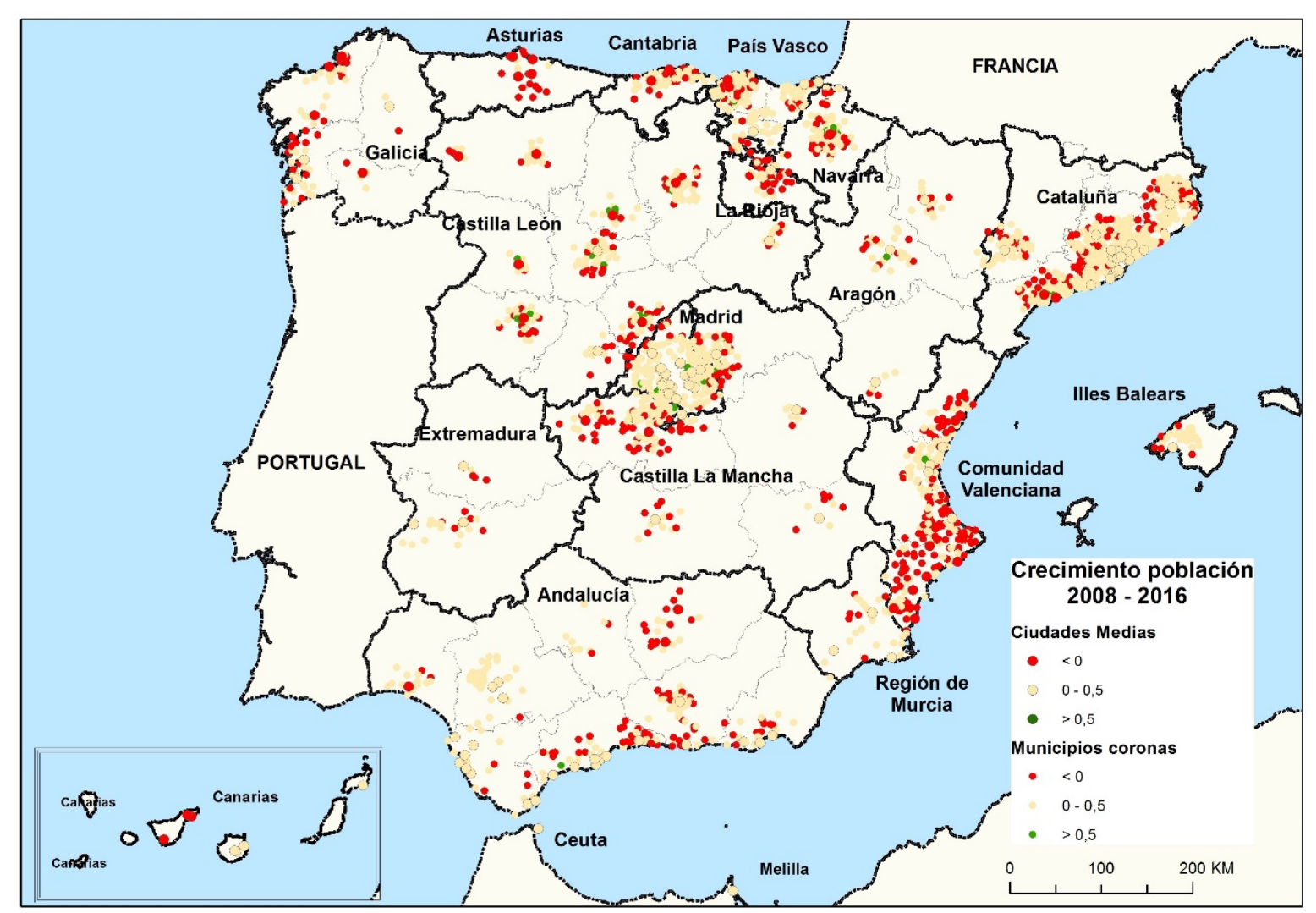

Figura 3 - Evolución de la población en las ciudades medias y sus coronas entre 2008 y 2016 . Fuente: Padrón Municipal de Habitantes (INE, 2018) y autores (2019).

Con el cambio de tendencia, 53 de las 136 ciudades medias españolas pierden población en el periodo de la crisis (un 39\%). Sin embargo, es necesario recordar que el conjunto de ciudades medias con sus respectivas coronas continúa ganando población durante la crisis, más de 900.000 habitantes en total. Em consecuencia, podemos afirmar que el grupo de las áreas urbanas formadas por las ciudades medias y los municipios dinámicos de sus coronas crece demográficamente durante la crisis a costa del resto del territorio, incluyendo a las grandes ciudades.

Sin embargo, durante la crisis encontramos varias áreas urbanas medias que tienen un saldo negativo global de población. Destacan en la escala regional el área urbana de Asturias (Rodríguez \& Carrero, 2013), que continúa su sangría demográfica, junto con el conjunto de las áreas urbanas de la Comunidad Valenciana, que pierden población de forma severa en el área metropolitana de Alicante y, en menor medida, en la de las ciudades medias de la provincia de Castellón. Otros ejemplos significativos son los de Jaén y Linares en Andalucía, y en Castilla y León, las áreas de León, Ponferrada, Salamanca, Segovia y Zamora.

\section{El desarrollo inmobiliario de las ciudades medias de España y sus coronas en el siglo XXI}

Los efectos del boom inmobiliario son evidentes en la evolución de las construcciones en las ciudades medias españolas y sus coronas. En lo que se ha llamado como un tsunami urbanizador (Fernández, 2006), los núcleos urbanos intermedios y sus áreas urbanas han recibido el impacto principal de la ola gigante de las nuevas construcciones. En la Tabla 5, los incrementos acumulados son muy significativos. Los datos incluyen nuevas edificaciones desde suelo, las mayoritarias, y obras nuevas sobre otras anteriormente derribadas, muchas menos en los núcleos urbanos españoles. Las ciudades medias españolas han aumentado sus unidades constructivas en un $43,69 \%$ sobre las edificaciones existentes en el 2000. Las coronas de estos núcleos urbanos disparan este incremento hasta el $61,33 \%$ con respecto al 
mismo año. Es decir, en lo que va del siglo XXI, las áreas urbanas de las ciudades medias españolas han experimentado un proceso de construcción y artificialización del suelo de casi dos terceras partes de lo que ya existía. El resto de los municipios de España también presentan porcentajes de incrementos constructivos elevados, pero con cifras menores a las de las ciudades medias y sus coronas.

Tabla 5 - Evolución de la construcción en España por categorías de análisis. 2000-2017

\begin{tabular}{|c|c|c|c|c|c|c|}
\hline \multirow{2}{*}{ Unidades constructivas } & \multicolumn{2}{|c|}{ Catastro 2000} & \multicolumn{2}{|c|}{ Catastro 2009} & \multicolumn{2}{|c|}{ Catastro 2017} \\
\hline & Total & $\%$ & Total & $\%$ & Total & $\%$ \\
\hline Muestra ciudades medias & 7.205 .230 & $28,67 \%$ & 2.879 .124 & $29,30 \%$ & 408.219 & $29,39 \%$ \\
\hline Coronas ciudades medias & 6.964 .858 & $27,72 \%$ & 3.485 .897 & $30,36 \%$ & 417.403 & $30,45 \%$ \\
\hline Resto de municipios & 10.959 .628 & $43,61 \%$ & 2.927 .173 & $40,34 \%$ & 448.607 & $40,16 \%$ \\
\hline Total & 25.129 .716 & $100 \%$ & 9.292 .194 & $100 \%$ & 1.274 .229 & $100 \%$ \\
\hline
\end{tabular}

Fuente: Catastro Inmobiliario (Espanha, 2018).

Hay una drástica diferencia entre el periodo del boom y el de la Gran Recesión. No obstante, en la actualidad sigue habiendo un crecimiento de las unidades constructivas del $4 \%$ en los núcleos urbanos intermedios y sus áreas urbanas (ver Tabla 6).

Tabla 6 - Porcentaje de crecimiento de la construcción en España por categorías de análisis. 2000-2017

\begin{tabular}{ccc}
\hline \% de crecimiento por periodo & $\mathbf{2 0 0 0 - 2 0 0 9}$ & $\mathbf{2 0 1 0 - 2 0 1 7}$ \\
\hline Muestra ciudades medias & $39,96 \%$ & $4,05 \%$ \\
Coronas ciudades medias & $50,05 \%$ & $3,99 \%$ \\
Resto de municipios & $26,71 \%$ & $3,23 \%$ \\
\hline
\end{tabular}

Fuente: Catastro Inmobiliario (Espanha, 2018).

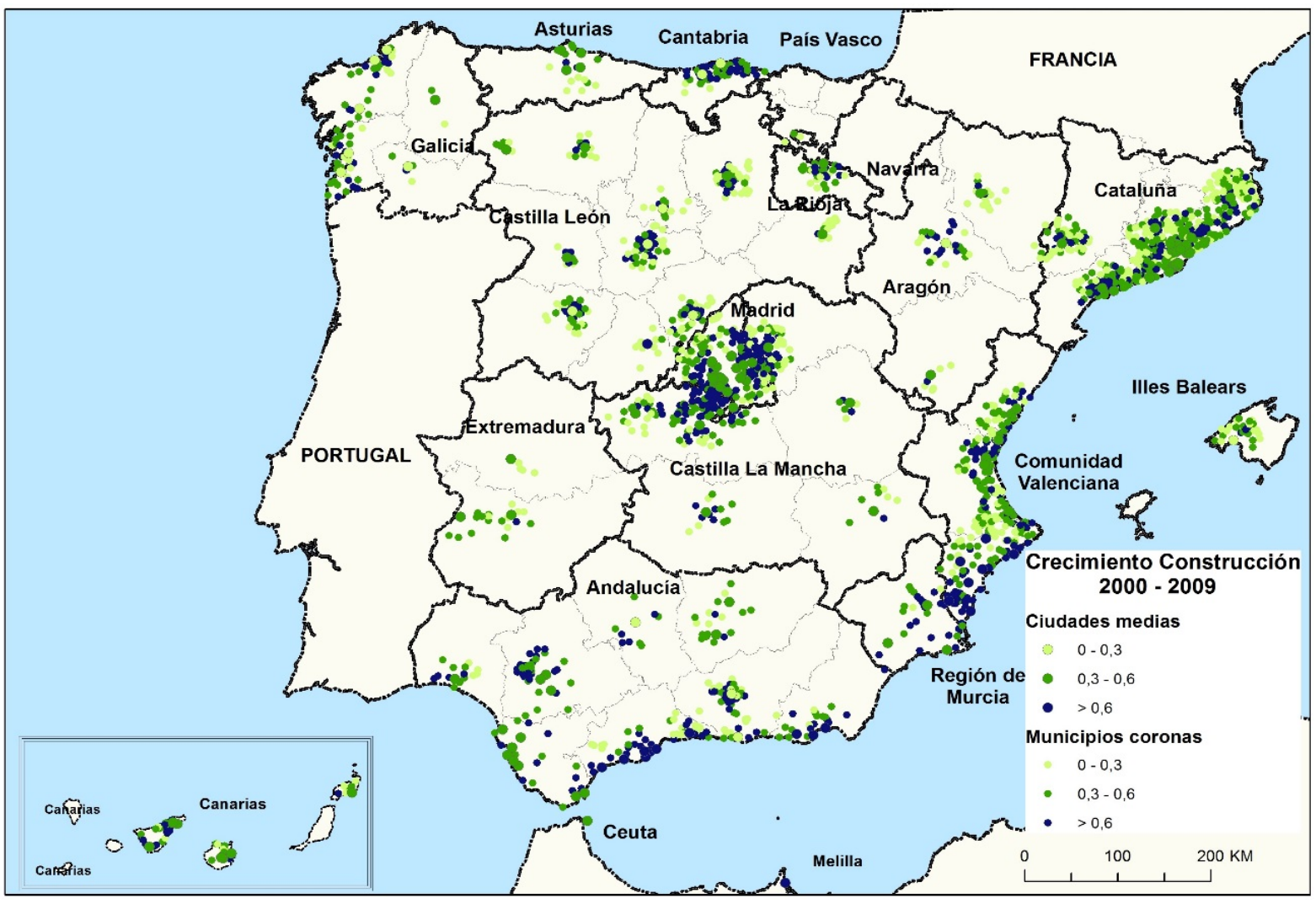

Figura 4 - Evolución de las unidades constructivas en las ciudades medias y sus coronas, 2000-2009. Fuente: Catastro Inmobiliario (Espanha, 2018) y autores (2019). 
En los años del incremento urbanístico explosivo, los municipios de las periferias tenían un incremento un 10\% superior al de los núcleos centrales. Ahora se pierde esta diferencia. En este sentido, el freno se produce en mayor medida en los municipios de las coronas, que pasan a crecer al mismo ritmo que las propias ciudades medias. La representación cartográfica del primer periodo entre los años $2000 \mathrm{y}$ 2009 (ver Figura 4) refleja los fuertes incrementos, predominando los colores verdes y azul oscuro en muchos municipios del área de estudio, fundamentalmente en las coronas de las ciudades medias. La dispersión de puntos refleja este hecho de manera geográfica coincidiendo con la disposición de la red urbana de España. Los espacios litorales, sobre todo en el Mediterráneo, y el área metropolitana de Madrid concentran la mayor parte de los núcleos de población

Se aprecian crecimientos moderados y sostenidos en la mayoría de los núcleos urbanos intermedios, que se ven reforzados con importantes aumentos en los municipios colindantes. Este patrón se reproduce por todo el país, siendo un fenómeno que se extiende por otros países europeos como han estudiado Lagarias \& Sayas (2018). Así, por ejemplo, los municipios de la corona de Guadalajara, ciudad media con un aumento similar a la media nacional, multiplican por veinte su parque constructivo haciendo que sea su área urbana la que más crece en conjunto, por encima de la segunda que es la correspondiente a las ciudades medias de las Comunidad de Madrid, verdadero motor del crecimiento especulativo en estos municipios (Roca et al., 2011).

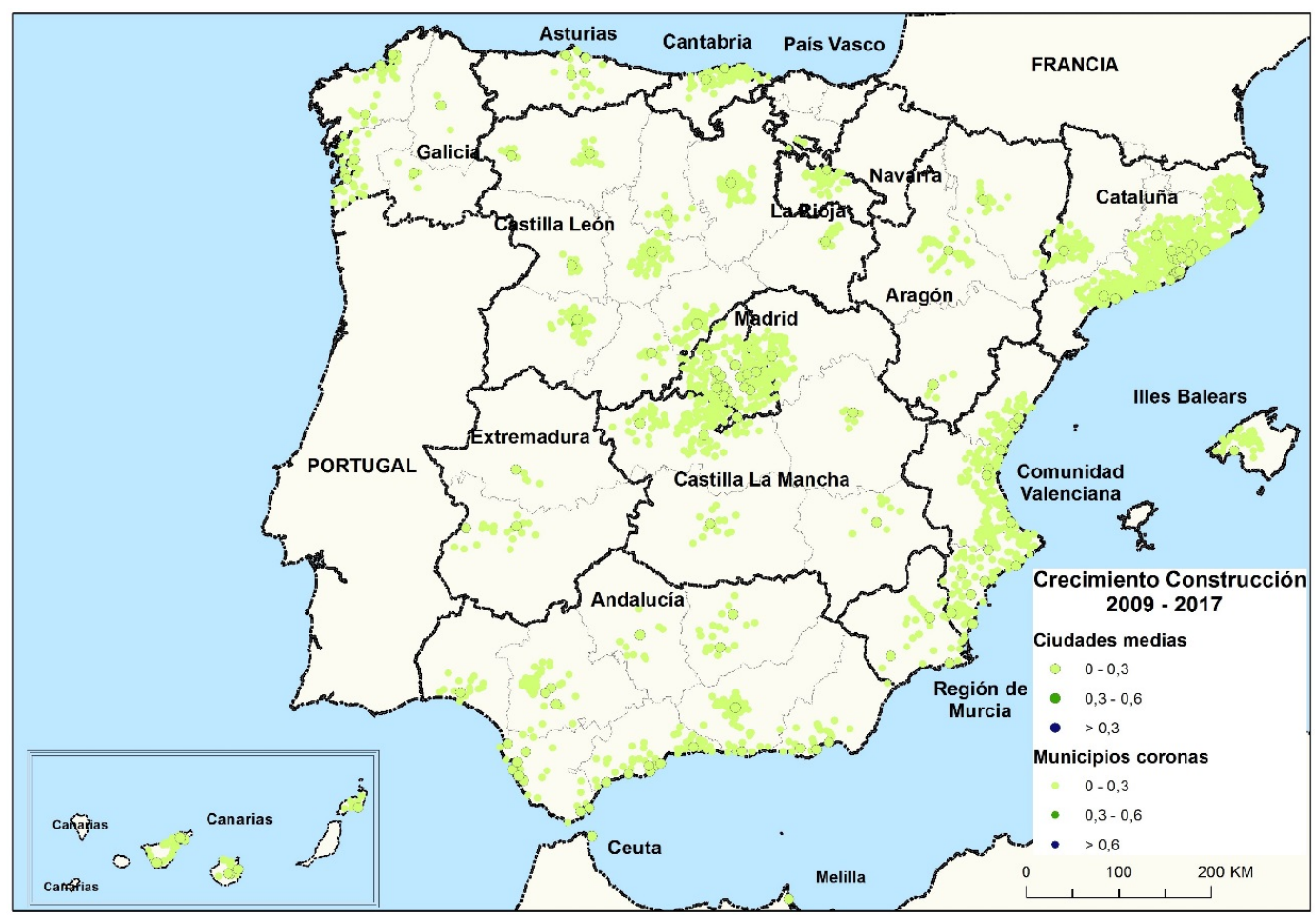

Figura 5 - Evolución de las unidades constructivas en las ciudades medias y sus coronas, 2010-2017. Fuente: Catastro Inmobiliario (Espanha, 2018) y autores (2019).

Por su parte son nuevamente las pequeñas capitales de provincia del noroeste y sus coronas, a las que se une también Teruel, con tasas de crecimiento más moderadas. También destacan en la época del boom los crecimientos relativamente moderados de las zonas más turísticas de nuestras costas

En el mapa de la evolución de las unidades constructivas en las ciudades medias y sus coronas, 2010-2017 (Figura 5), todos los asentamientos representados se mueven en una horquilla de incremento constructivo entre el $0 \%$ y el $0,3 \%$. El número de unidades constructivas nuevas se ha reducido drásticamente con respecto al periodo anterior estudiado (2000-2009). La uniformidad es absoluta y viene a reflejar el parón que sufrió España con la crisis en el sector de la construcción. El mapa por sí solo dice poco, pero es impactante al compararlo con el de 2000-2009. 


\section{Relación entre el crecimiento de población y construcciones para las ciudades medias y sus coronas en áreas urbanas metropolitanas y no metropolitanas}

El cuadro de síntesis (ver Tabla 7) refleja de forma clara las tendencias ya detectadas de crecimiento diferenciado para las ciudades medias y los municipios de sus coronas dentro y fuera de las áreas metropolitanas. Se muestra, como los porcentajes de crecimiento en el número de construcciones son siempre superiores a los del aumento de población.

Tabla 7 - Cuadro de síntesis de crecimiento comprado de población y construcciones para áreas urbanas metropolitanas y no metropolitanas

\begin{tabular}{ccccc}
\hline & \multicolumn{2}{c}{ Crecimiento población } & \multicolumn{2}{c}{ Crecimiento construcciones* } \\
\hline No Metropolitanas & $\mathbf{2 0 0 0 - 2 0 0 8}$ & $\mathbf{2 0 0 9 - 2 0 1 6}$ & $\mathbf{2 0 0 0 - 2 0 0 9}$ & $\mathbf{2 0 1 0 - 2 0 1 7}$ \\
Ciudades medias & $7 \%$ & $0 \%$ & $35 \%$ & $14 \%$ \\
Municipios coronas & $20 \%$ & $3 \%$ & $47 \%$ & $13 \%$ \\
Metropolitanas & $\mathbf{2 0 0 0 - 2 0 0 8}$ & $\mathbf{2 0 0 9 - 2 0 1 6}$ & $\mathbf{2 0 0 0 - 2 0 0 9}$ & $\mathbf{2 0 1 0 - 2 0 1 7}$ \\
Ciudades medias & $20 \%$ & $4 \%$ & $43 \%$ & $14 \%$ \\
Municipios coronas & $26 \%$ & $5 \%$ & $53 \%$ & $11 \%$ \\
Diferencia & $\mathbf{2 0 0 0 - 2 0 0 8}$ & $\mathbf{2 0 0 9 - 2 0 1 6}$ & $\mathbf{2 0 0 0 - 2 0 0 9}$ & $\mathbf{2 0 1 0 - 2 0 1 7}$ \\
Ciudades medias & $13,02 \%$ & $3,46 \%$ & $8,80 \%$ & $0,00 \%$ \\
Municipios coronas & $6,78 \%$ & $1,13 \%$ & $6,11 \%$ & $-1,34 \%$ \\
\hline
\end{tabular}

*Excepto País Vasco y Navarra. Fuente: Catastro Inmobiliario (Espanha, 2018) y Padrón Municipal de Habitantes (INE, 2018).

Los crecimientos de población durante los dos periodos son más intensos para las ciudades medias metropolitanas y sus coronas, pero la diferencia entre las ciudades medias es mucho mayor que entre las coronas, prácticamente el doble tanto durante el boom como durante la crisis. Con respecto a las construcciones, las diferencias son menos acusadas durante la fase expansiva, pero en la crisis desaparecen en las ciudades medias mientras que para los municipios de las coronas se invierte la tendencia, pasando a crecer de forma ligeramente superior en las no metropolitanas. El crecimiento comparado entre población y construcciones, aumenta de forma más ajustada en las ciudades de las áreas metropolitanas en los dos periodos y también en sus coronas

Para un acercamiento cuantitativo más ajustado se ha aplicado a las dos variables el coeficiente de correlación de Pearson. Su valor varía entre -1 y 1 y se considera que hay una correlación positiva cuanto más próximo sea a 1 (Cohen et al., 2003). La correlación es muy significativa para los dos periodos de estudio para el conjunto de todas las ciudades medias; durante el boom económico para la agregación de ciudades y coronas que forman las áreas urbanas metropolitanas, así como para el conjunto de los municipios de todas las coronas y solo es significativa para estos últimos en la época de la crisis.

Encontramos una correlación solo significativa para los municipios del resto del territorio en el boom, relación que sin embargo pasa a carecer cualquier significación desde 2009, (ver Tabla 8) cuestión lógica pues mientras las construcciones crecen, aunque muy poco, la población directamente decrece. Finalmente, en las áreas urbanas no metropolitanas la correlación es poco significativa para el mismo periodo, cuando se invierte la tendencia y las construcciones crecen en mayor medida.

Tabla 8 - Coeficientes de correlación entre crecimiento de población y de construcciones en datos absolutos*

\begin{tabular}{ccc}
\hline & $\mathbf{2 0 0 0 - 2 0 0 8 / 9}$ & $\mathbf{2 0 0 9 / 1 0 - 2 0 1 6 / 1 7}$ \\
\hline Ciudades medias & 0,99 & 0,92 \\
Municipios de las coronas & 0,92 & 0,62 \\
Municipios fuera de las áreas urbanas & 0,70 & 0,03 \\
Áreas urbanas metropolitanas & 0,92 & 0,74 \\
Áreas urbanas no metropolitanas & 0,75 & 0,37 \\
\hline
\end{tabular}

*Excepto País Vasco y Navarra. Fuente: Catastro Inmobiliario (Espanha, 2018) y Padrón Municipal de Habitantes (INE, 2018).

Sin embargo, la correlación casi perfecta (0.99) se produce en el conjunto de todas las ciudades medias pese a que algunas de ellas decrecen. Esta significación refuerza la idea de que los procesos de edificación en muchos de los municipios de las coronas son de carácter especulativo ya que en ellos aumenta la población porque previamente se ha incrementado la oferta de vivienda. 
Finalmente, se ha realizado un gráfico de dispersión relacionando el porcentaje de crecimiento de la población con el porcentaje de crecimiento de las viviendas en las coronas de las ciudades medias. Dada la complejidad de la representación se ha considerado agrupar las coronas por las provincias en las que se encuentran. El cambio de escala de análisis puede llegar a ocultar casos de coronas con solapamientos en dos provincias. Sin embargo, el gráfico presenta de forma clara diferentes comportamientos en los distintos territorios de España (ver Figura 6).

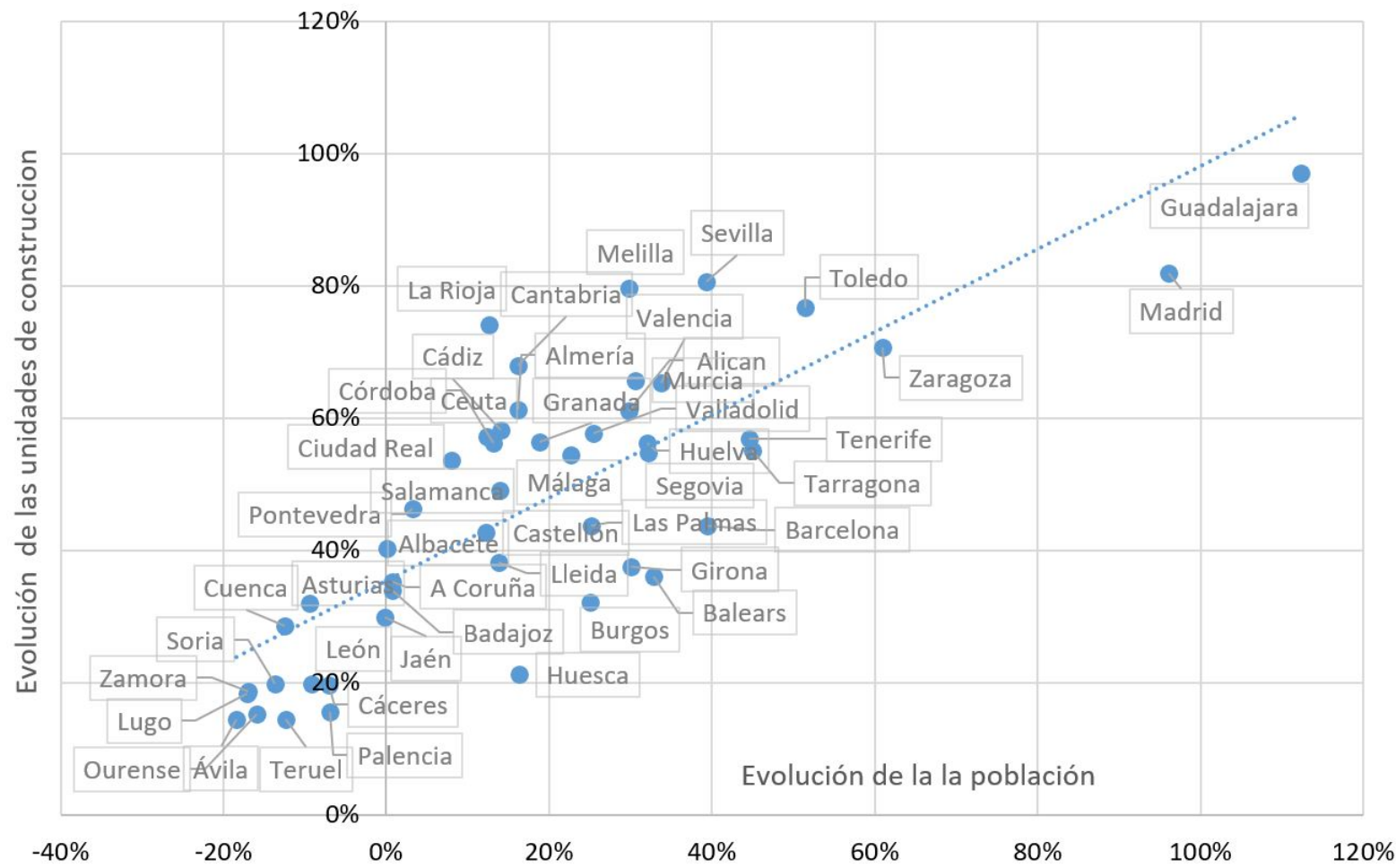

Figura 6 - Gráfico de dispersión entre el crecimiento de la población y la construcción de viviendas (2000-2018) en las ciudades medias españolas por provincias. Fuente: Catastro Inmobiliario (Espanha, 2018), Padrón Municipal de Habitantes (INE, 2018). Autores (2019).

El gráfico presenta de una forma clara el caso extremo de Madrid que llega a superar sus límites provinciales a través del corredor del Henares en la provincia de Guadalajara y de la comarca de la Sagra en la provincia de Toledo. Destaca igualmente el caso de Zaragoza (Escolano et al., 2018).

En el lado opuesto, encontramos un claro ejemplo de la urbanización difusa en las ciudades medias en sus coronas por los efectos del crecimiento inmobiliario de los 2000 . Se trata de las periferias de ciudades medias con crecimientos moderados (no superiores al 30\%). Se sitúan en capitales provinciales cuyo tamaño demográfico se halla en la parte baja del rango que las define, y con una distribución espacial en la mitad norte de la península, especialmente en el sector occidental, tal y como se podía ya intuir en los mapas del primer periodo estudiado.

\section{Conclusiones}

Las dinámicas demográficas y el desarrollo inmobiliario de las ciudades medias de España y sus coronas entre 2000 y 2017 se organizan en dos grandes fases que han tenido la evolución demográfica y constructiva en el país. Una primera, hasta el 2008, caracterizada por el aumento de la población y por un incremento explosivo de la construcción y una segunda posterior con una paralización de ambas. Em la primera fase, en las ciudades medias y, especialmente en sus coronas, se aplicó activamente el modelo de urbanización disperso fundamentado en la idea de que la ciudad es una "máquina de 
crecimiento" (grow machine). De esta forma. una alianza entre diferentes agentes urbanos, los gobiernos locales, los propietarios del suelo, las empresas constructoras y promotoras, el sector financiero y un amplio sector de los ciudadanos (con las lógicas diferencias locales entre ciudades que no se abordan aquí), dio lugar a un proceso de urbanización especulativo, explosivo y disperso. El resultado fue una inmensa burbuja inmobiliaria que en las ciudades medias de España y sus coronas estalla en 2008 y da lugar a una fase de paralización absoluta con graves implicaciones económicas, crisis de todo el sector constructivo y de los bancos; políticas, déficits públicos de los ayuntamientos; y sociales, devaluación de las viviendas y, en los peores casos, desahucios derivados del impago de las hipotecas. La Gran Recesión también ha supuesto graves consecuencias sobre el territorio pues el modelo se traduce en áreas urbanas con un elevado grado de insostenibilidad tanto por los costes de los desplazamientos, infraestructuras y servicios repartidos ahora en espacios urbanos muy dispersos, como por el impacto medioambiental.

No obstante, tras el análisis realizado, se constata que el cambio de ritmo en el crecimiento de población y construcciones es bastante diferenciado geográficamente. El efecto gravitacional que en décadas anteriores había caracterizado al sistema urbano español, concentrando en la periferia de las grandes ciudades industriales o turísticas el mayor crecimiento urbano, pasa durante el periodo del boom a caracterizar al conjunto de las ciudades medias. Por lo tanto, se convierte en una tendencia independiente al tamaño de la ciudad, media o grande, y también de los límites administrativos, pues en el caso más extremo, Madrid, traspasa su comunidad autónoma. Además, tiene en general un cierto efecto centrífugo, ya que el crecimiento de las coronas frena el incremento de la población de la ciudad central, que en muchos casos pierde población, aunque el conjunto del área la gane. Finalmente, se aprecia que fuera de las grandes metrópolis, las áreas urbanas de las ciudades medias capitales de provincia, donde no hay otros núcleos urbanos similares, resisten mejor la crisis que las situadas en provincias con varias ciudades medias. El artículo aporta un análisis actualizado sobre las dinámicas más recientes que han seguido las ciudades medias españolas y sus coronas. Los indicadores actuales nos señalan que los crecimientos explosivos constructivos, así como los incrementos demográficos notables no se producirán a corto plazo. En investigaciones futuras, siguiendo la senda abierta por este trabajo, se deberán continuar analizando las evoluciones demográficas y de las unidades constructivas en las ciudades medias en España, dada la importancia de ambos procesos. Queda pendiente la ampliación con otros datos como la ocupación del suelo, al igual que la necesaria complementación con estudios de casos para la comprensión de los factores locales, y cómo han afectado a las dinámicas generales aquí presentadas en cada área urbana intermedia española en concreto.

\section{Agradecimientos}

Nos gustaría agradecer a los editores la revista Urbe. Revista Brasileira de Gestão Urbana y a los revisores anónimos sus comentarios para nuestro manuscrito. Su contribución ha mejorado notablemente el artículo. Los autores quieren agradecer al profesor Francisco Cebrián Abellán por introducirnos en la investigación de las ciudades medias.

\section{Referencias}

Abellán, G. A., Moreno, J. A., \& Vinuesa, A. J. (1978). Propuesta de tipología para ciudades españolas de tipo medio. Estudios Geográficos, 152, 285-306. Recuperado el 19 de julio de 2019, de https://repositorio.uam.es/handle/10486/672622

Adam, B. (2006). Medium-sized cities in urban regions. European Planning Studies, 14(4), 547-555. http://dx.doi.org/10.1080/09654310500421220.

Andrés, G. (2008). Geografía y ciudades medias en España: ¿A la búsqueda de una definición innecesaria? Scripta Nova: Revista Electrónica de Geografía y Ciencias Sociales, 12(270), 49. Recuperado el 19 de julio de 2019, de http://www.ub.edu/geocrit/sn/sn-270/sn-270-49.htm 
Angeoletto, F., Santos, J. W. M. C., Ruiz Sanz, J. P., Silva, F. F., \& Albertín, R. M. (2016). Tipología socio-ambiental de las ciudades medias de Brasil: aportes para un desarrollo urbano sostenible. Urbe. Revista Brasileira de Gestão Urbana, 8(2), 272-287. http://dx.doi.org/10.1590/2175-3369.008.002.A008.

Bellet, C. (2012). Las ciudades intermedias en los tiempos de la globalización. In M. Llop, \& E. Usón (Eds.), Ciudades intermedias: dimensiones y definiciones (pp. 222-251). Lleida: Milenio.

Bellet, C., \& Gutiérrez, A. (2015). Los efectos territoriales del crecimiento y la producción inmobiliaria en ciudades medias españolas: el caso de Lleida (1990-2012). In C. Bellet, E. Melazzo, M. E. Sposito, \& J. M. Llop (Eds.), Urbanización, producción y consumo en ciudades medias/intermedias (pp. 297-314). Lleida: Universitat de Lleida.

Bellet, C., \& Olazábal, E. (2017). Las ciudades intermedias en España: dinámicas y procesos de urbanización recientes. In F. Maturana, M. E. Sposito, C. Bellet, C. Henríquez, \& F. Arenas (Eds.), Sistemas urbanos y ciudades medias en Iberoamérica (pp. 146-185). Santiago de Chile: GEOlibros.

Campos Sánchez, F. S. (2017). Ciudades medias españolas siglo XXI: revisión y análisis bibliométrico de enfoques y temáticas predominantes. Cuadernos Geográficos, 56(1), 217-241. Recuperado el 19 de julio de 2019, de http://revistaseug.ugr.es/index.php/cuadgeo/article/view/3960/5316

Cano, G. (2008). Clasificaciones urbanas en Andalucía: las ciudades medias. Revista de Estudios Andaluces, 27(27), 115153. http://dx.doi.org/10.12795/rea.2008.i27.05. [de https://idus.us.es/xmlui/handle/11441/13440]

Cebrián, F. (2007). Ciudades con límites y ciudades sin límites: manifestaciones de la ciudad difusa en Castilla-La Mancha. Boletín de la Asociación de Geógrafos Españoles, 262, 221-240. Recuperado el 19 de julio de 2019, de https://www.age-geografia.es/ojs/index.php/bage/article/view/589

Clayton, N., \& Morris, K. (2010). Recession, recovery and medium-sized cities. London: The Work Foundation.

Cohen, J., Cohen, P., West, S. G., \& Aiken, L. S. (2003). Applied multiple regression/correlation analysis for the behavioral sciences (3rd ed.). London: Lawrence Earlbaum Associates.

De Cos, O., \& De Meer, Á. (2013). Las áreas metropolitanas de tamaño medio: la configuración de un espacio de cohesión en el conjunto polinuclear Santander-Torrelavea. Ciudad y Territorio: Estudios Territoriales, 45(146), 351-362. Recuperado el 19 de julio de 2019, de https://www.xiiicongresodelapoblacionespanola.unican.es/images/Actas.pdf

De Ureña, J. M. A., Pillet, F., \& Marmolejo, C. (2013). Aglomeraciones urbanas basadas en varios centros: el policentrismo. Ciudad y Territorio: Estudios Territoriales, 45(176), 249-266. Recuperado el 19 de julio de 2019, de https://www.xiiicongresodelapoblacionespanola.unican.es/images/Actas.pdf

Escolano, S., López, C., \& Pueyo, Á. (2018). Urbanismo neoliberal y fragmentación urbana: el caso de Zaragoza (España) en los primeros quince años del siglo XXI. EURE: Revista Latinoamericana de Estudios Urbano Regionales, 44(132), 183210. http://dx.doi.org/10.4067/s0250-71612018000200185.

Escudero Gomez, L. A., \& Somoza Medina, J. (2010). Medium-sized cities: polycentric strategies vs the dynamics of metropolitan area growth. The Open Urban Studies Journal, 3(1), 2-13.

http://dx.doi.org/10.2174/1874942901003010002.

Espanha. Dirección General del Catastro. (2018). Estadísticas catastrales. Recuperado el 19 de julio de 2019, de http://www.catastro.meh.es/esp/estadisticas.asp

Esteban, A., \& López, A. (1989). El papel de las ciudades medias en España: presente y futuro. Urbanismo, 6, 6-16. Recuperado el 19 de julio de 2019, de https://www.coam.org/media/Default\%20Files/fundacion/biblioteca/revistaurbanismo/docs/revista-urbanismo-n6-pag6-16.pdf

Feria, J., \& Albertos, J. M. (2010). La ciudad metropolitana en España, procesos urbanos en los inicios del siglo XXI. Cizur Menor: Thomson Reuters-Civitas.

Fernández, D., \& Corbelle, E. (2017). Cambios en los usos de suelo en la Península Ibérica: un meta-análisis para el período 1985-2015. Biblio3W: Revista Bibliográfica de Geografía y Ciencias Sociales, 22(125). Recuperado el 19 de julio de 2019, de http://www.ub.edu/geocrit/b3w-1215.pdf

Fernández, R. (2006). El tsunami urbanizador español y mundial. Barcelona: Virus Editorial.

Gaja, F. (2008). El "tsunami urbanizador” en el litoral mediterráneo. El ciclo de hiperproducción inmobiliária 1996-2006. Scripta Nova: Revista Electrónica de Geografía y Ciencias Sociales, 12(270). Recuperado el 19 de julio de 2019, de http://www.ub.es/geocrit/sn/sn-270/sn-270-66.htm 
Galeano, E. (1998). Patas arriba: la escuela del mundo al revés. Madrid: Siglo XXI.

Ganau, J., \& Vilagrasa, J. (2003). Ciudades medias en España: posición en la red urbana y procesos urbanos recientes. In H. Capel (Ed.), Mediterráneo económico: ciudades, arquitectura y espacio urbano. Bareslona: Cajamar Caja Rural.

Garrido-Jiménez, F. J., Magrinyá-Torner, F., \& Consuelo del Moral-Ávila, M. (2017). The relative length of roads and housing density as distinct determinants of operating costs for urban services: evidence from medium-sized Spanish cities. Journal of Urban Technology, 24(1), 75-91. http://dx.doi.org/10.1080/10630732.2016.1177272.

Gutiérrez, A., \& Paül, D. (2012). Las ciudades intermedias como objeto de estudio, una categorización de las diferentes aproximaciones. In J. M. Llop, \& E. Usón (Eds.), Ciudades intermedias: dimensiones y definiciones (pp. 354-383). Lleida: Milenio.

Hildreth, P. A. (2006). Roles and economic potential of English medium-sized cities: a discussion paper. Manchester: University of Salford. Recuperado el 19 de julio de 2019, de https://www.researchgate.net/publication/228379144_Roles_and_Economic_Potential_of_English_MediumSized_Cities_A_Discussion_Paper

Indovina, F. (1998). Algunes consideracions sobre la "ciutat difusa”. Documents d'Anàlisi Geogràfica, 33, 21-32. Recuperado el 19 de julio de 2019, de https://www.raco.cat/index.php/DocumentsAnalisi/article/view/31661

Instituto Nacional de Estadística - INE. (2018). Padrón municipal de habitantes. Recuperado el 19 de julio de 2019, de https://www.ine.es/dyngs/INEbase/es/operacion.htm?c=Estadistica_C\&cid=1254736177012\&menu=resultados\&secc $=1254736195461 \& \mathrm{idp}=1254734710990$

Lagarias, A., \& Sayas, J. (2018). Urban sprawl in the mediterranean: evidence form coastal medium-sized cities. . Regional Science Inquiry, 10(3), 15-32. Recuperado el 19 de julio de 2019, de https://ideas.repec.org/a/hrs/journl/vxy2018i3p15-32.html

Llop, J. M., \& Usón, E. (Eds.). (2012). Ciudades intermedias: dimensiones y definiciones. Lleida: Milenio.

Llorente del Río, A. (2010). Transformaciones territoriales metropolitanas en las ciudades medias. Cambios de modelo urbano bajo los procesos de la globalización: el caso de Santander. In C. Cornejo, J. Morán Sáez, \& J. P. Trigo (Eds.), Ciudad, territorio y paisaje: reflexiones para un debate multidisciplinar (pp. 50-62). Madrid: CSIC.

López Trigal, L. (2014). Identificación y caracterización estratégica de las ciudades y aglomeraciones medias ibéricas. Ciudad y Territorio. Estudios Territoriales, 182, 625-648. Recuperado de https://dialnet.unirioja.es/servlet/ejemplar?codigo=382734\&info=open.

Mallarach, J., \& Vilagrasa, J. (2002). Los procesos de descentralización urbana en las ciudades medias españolas. Eria, 57, 57-70. http://dx.doi.org/10.17811/er.2.2018.183-203.

Marinovic-Uzelac, A. (1999). Middle towns: myth or reality? Drustvena Istrazivanja. Journal for General Social Issues, 8(1), 3-20. Recuperado el 19 de julio de 2019, de https://hrcak.srce.hr/31473

Marques da Costa, E. (2002). Ciudades médias: contributos para a sua definição. Finisterra: Revista Portuguesa de Geografia, 37(74), 101-128. Recuperado el 19 de julio de 2019, de https://www.researchgate.net/publication/28180780_Ciudades_medias_contributos_para_a_sua_definicao

Maturana, F., \& Rojas, A. (Eds.). (2016). Ciudades intermedias en Chile: territorios olvidados. Santiago de Chile: RIL Editores.

Méndez, R. (2010). La dimensión urbana del desarrollo territorial: significado actual de las ciudades de tamaño intermedio y las periferias metropolitanas. In C. Cornejo, J. Morán Sáez, \& J. P. Trigo (Eds.), Ciudad, territorio y paisaje: reflexiones para un debate multidisciplinar (pp. 137-155). Madrid: CSIC.

Moyart, L. (2005). The role of producer services in regional development: what opportunities for medium-sized cities in Belgium. Service Industries Journal, 25(2), 213-228. http://dx.doi.org/10.1080/0264206042000305420.

Obrębalski, M. (2017). Demographic potential in functional areas of the selected medium-sized cities in Poland and the Czech Republic. Geoscape, 11(1), 16-24. http://dx.doi.org/10.1515/geosc-2017-0002.

Pérez, Y., \& Rojas, Y. (2017). Estrategias de manejo y resolución de conflictos socio-ambientales relacionados con los efectos del cambio climático en ciudades intermedias de América Latina y el Caribe. In N. Novillo, P. Olmedo M., Y. Pérez, \& Y. Rojas Paiva (Eds.), Aproximaciones al estudio de la relación entre ciudades y cambio climático (pp. 111). Ecuador: FLACSO. Recuperado el 19 de julio de 2019, de https://flacso.edu.ec/cambioclimatico/wpcontent/uploads/2018/10/Cambio-climatico-ciudades-genero-1.pdf\#page=112 
Pillet, F. (2008). El catastro inmobiliario territorial de urbana y rústica en España. Scripta Nova: Revista Electrónica de Geografía y Ciencias Sociales, 12(274). Recuperado el 19 de julio de 2019, de http://www.ub.es/geocrit/sn/sn-274.htm

Precedo, A., \& Míguez, A. (2014). Las ciudades medias de la globalización. Madrid: Síntesis.

Reher, D. S., \& Valero Lobo, A. (2005). Fuentes de información demográfica en España (3. ed.). Madrid: CSIC.

Reques, P., \& De Cos, O. (2012). La población en clave territorial: procesos, estructuras y perspectivas de análisis. In Actas del XIII Congreso de la Población Española. Santander: Ministerio de Economía y Competitividad. Recuperado el 19 de julio de 2019, de https://www.xiiicongresodelapoblacionespanola.unican.es/images/Actas.pdf

Roca, J., Arellano, B., \& Moix, M. (2011). Estructura urbana, policentrismo y “sprawl”: los ejemplos de Madrid y Barcelona. Ciudad y Territorio: Estudios Territoriales, (168), 299-321. Recuperado el 19 de julio de 2019, de https://upcommons.upc.edu/bitstream/handle/2117/13579/04_CyTET_168web.pdf

Roca, J., Moix, M., \& Arellano, B. (2012). El sistema urbano en España. Scripta Nova: Revista Electrónica de Geografía y Ciencias Sociales, 15(396). Recuperado el 19 de julio de 2019, de http://www.ub.edu/geocrit/sn/sn-396.htm

Rodríguez, F., \& Carrero, M. (2013). Ciudad Astur, una singularidad metropolitana. Ciudad y Territorio: Estudios Territoriales, 45(176), 333-350. Recuperado el 19 de julio, de https://www.xiiicongresodelapoblacionespanola.unican.es/images/Actas.pdf

Salom, J. (1995). Ciudades medias y centros intermedios en el Arco Mediterráneo. In A. Campesino, M. Á. Troitiño Vinuesa, \& M. L. Campos Romero (Eds.), Las ciudades españolas a finales del siglo XX (pp. 53-56). Cuenca: AGE.

Servillo, L., Atkinson, R., \& Hamdouch, A. (2017). Small and medium-sized towns in Europe: Conceptual, methodological and policy issues. Tijdschrift voor Economische en Sociale Geografie, 108(4), 365-379.

http://dx.doi.org/10.1111/tesg.12252.

Somoza Medina, J. (2013). Gobernanza urbana, competitividad y procesos de renovación en las ciudades medias españolas. Boletín de la Asociación de Geógrafos Españoles, 61, 47-66. http://dx.doi.org/10.21138/bage.1535.

Vilagrasa, J. (1999). Las ciudades pequeñas y medias en España. In R. Domínguez (Ed.), La ciudad: tamaño y crecimiento (pp. 17-49). Málaga: AGE.

Vilagrasa, J. (2000). Ciudades medias y ciudades intermedias: posicionamiento en la red urbana y procesos urbanos recientes. Lleida: Universitat de Lleida.

Vinuesa, J. (1989). La población de las ciudades medias españolas. Urbanismo, 6, 17-27.

Zúñiga, I. (2016). La forma del límite en las ciudades medias españolas. Cuadernos de Investigación Urbanística, (105), 166. Recuperado el 19 de julio de 2019, de http://polired.upm.es/index.php/ciur/article/view/3450

Editor: Paulo Nascimento Neto

Recibido: Jul. 25, 2019

Aprobado: Oct. 21, 2019 